\title{
Ophthalmologica
}

D.R. Korb, J. Craig, M. Doughty, J.-P. Guillon, G. Smith, A. Tomlinson

\section{The Tear Film}

Structure, Function and Clinical Examination

Butterworth-Heinemann, 2002

214 pp.; GBP 45.00

ISBN 0-7506-4196-7

Dieses von sechs ausgewiesenen Experten des internationalen englischsprachigen Raumes verfasste Buch spiegelt das derzeitige Wissen über das Krankheitsbild der Keratokonjunktivitis sicca (KCS) wider. Es stellt die verschiedenen Aspekte des Tränenfilms dar und seine Interaktionen und Beziehungen zu Bindehaut, Hornhaut und den Lidfunktionen.

Auch Alterungsprozesse des Tränenfilms, pathologische Veränderungen und einige Behandlungsstrategien werden diskutiert, wobei der Leitgedanke, dass dieses Buch primär dem Praktiker und nicht dem wissenschaftlich arbeitenden KCS-Forscher dienen soll, konsequent durchgehalten wird. Bei den vielen verschiedenen diagnostischen Möglichkeiten der KCS werden die wichtigsten dargestellt und mit Vor- und Nachteilen diskutiert.

Es gibt bereits über 40 labortechnische Untersuchungsmöglichkeiten von denen ebenfalls nur die in der Praxis eventuell anwendbaren ausgewählt wurden. Betont wird dabei, dass nie ein einziger Test zur Sicherung ausreicht, sondern ein Diagnose-Puzzle erforderlich ist.

Am besten bewährt hat sich im Arbeitsalltag noch immer die Kombination aus subjektivem Beschwerdebild, der break-up-time (BUT) und dem Schirmer Test. Wichtig ist dabei der Hinweis, dass die BUT keinesfalls mit kommerziell vorgefertigten Papierstreifen durchgeführt werden darf, sondern ausschliesslich mit flüssigem 2\%igem Fluoreszein $(10 \mu 1)$. Die Farbe löst sich gerade bei KCS oft nicht ausreichend gut aus den Papierstreifen, welche ausserdem oft noch an der trockenen Bindehaut festkleben.

Gut ist die Zusammenstellung der häufig gebrauchten Medikamentengruppen, die zur KCS führen (Kapitel 6, Seite 175) bzw. auch die Aufstellung systemischer Erkrankungen, die praktisch immer mit einer KCS einhergehen. Ausführlich wird dabei besonders auf das Sjögren-Syndrom eingegangen und die modernen Therapiemöglichkeiten mit lokalem Cyclosporin A erwähnt. Darüber hinaus werden im gesamten Buch praktisch keine konkreten Therapieempfehlungen gegeben, ausser z.B. Lidkantenhygiene bei Blepharitiden. Ein abschliessender Therapieteil, der sich auf die verschiedenen Ursachen und Störungsmuster des Tränenfilms bezieht, fehlt komplett.

Das Buch endet mit einem Kapitel über die Bedeutung des Tränenfilms heute und morgen und einer subjektiven Aufstellung des Herausgebers, wie er im praktischen Alltag diagnostisch an Tränenfilmstörungen herangeht.

Jedem der sechs Kapitel ist ein sich auf das Thema beziehendes und nicht allzu ausführliches, aber relevantes Literaturverzeichnis angehängt, so dass besonders Interessierte damit weiterarbeiten können.
Insgesamt handelt es sich um ein gut lesbares Buch, das wissenschaftlich nicht überfrachtet ist und im wesentlichen heutiges Grundlagenwissen widerspiegelt.

Es ist ein sehr gutes Buch für den Praxisalltag, in dem die KCS ja inzwischen einen breiten Raum einnimmt. Seinem Wunsch dem Praktiker zu dienen kommt es weitgehend nach, wenngleich es wünschenswert gewesen wäre, dass abschliessend ein ausführliches Therapiekapitel angefügt worden wäre, das Bezug auf die verschiedenen Ursachen der KCS nimmt und Störungen der verschiedenen Tränenfilmschichten respektiert.

Das Buch ist jedem Kollegen, besonders denen die mit Kontaktlinsen arbeiten, sehr zu empfehen.

I. Strempel, Marburg

\section{J. Knaus, Ph. Hugger \\ Augenheilkunde CD-ROM: Kompendium für Klinik und Praxis}

2., neu bearbeitete und erweiterte Aufl.

Deutscher Ärzteverlag, Köln

ISBN 3-7691-1173-7

Mit der vorliegenden CD-ROM werden nunmehr in 2., erweiterter Auflage die gängigen Untersuchungstechniken, Krankheitsbilder und Operationsverfahren der Augenheilkunde in kompakter Weise dargestellt. Es wird ein Überblick über die Geschichte der Augenheilkunde sowie die Anatomie und Physiologie des Sehorgans gegeben. Ferner enthält die CD-ROM die Weiterbildungsordnung für das Fach Augenheilkunde und die für die Fallpauschalenkodierung notwendige aktuelle Version des ICD-10 und des OPS-301.

Die knapp gehaltene Textgestaltung entspricht der eines Nachschlagewerkes. Mit Hilfe einer Suchfunktion und der Darstellung der Kapitelüberschriften in einem separaten Bildschirmfenster wird die Suche nach der gewünschten Information erleichtert. Der Informationszugriff ist ähnlich schnell wie bei einem Printmedium. Die Handhabung gestaltet sich einfach.

In über 800 Abbildungen in meist guter Qualität werden typische Befunde, Operationsverfahren und -instrumentarien gezeigt. Ein weiterer Einblick in die Techniken der Augenheilkunde wird durch sechs Videosequenzen gewährt, in denen Operationsverfahren wie die Goniotrepanation, Phakoemulsifikation, Pars-plana-Vitrektomie, LASIK, und die perforierende Keratoplastik gezeigt werden. Die Bildqualität der Videos befriedigt allerdings nicht immer. Auch erfolgt die Wiedergabe ohne erläuternden Kommentar.

Durch Verknüpfungen zu Informationen des Internets nutzen die Autoren einen Vorteil der Darstellung medizinischer Sachverhalte in der gewählten digitalen Form. Ein weiterer Ausbau der Möglichkeiten der multimedialen Darstellung wäre wünschenswert.

Alles in allem eine geeignete Informationsquelle, um den Studenten und Berufsanfänger in das Arbeitsfeld und die Praxis des Augenarztes einzuführen, aber auch dem Praktiker eine Hilfestellung $\mathrm{zu}$ leisten.

Ch. Meltendorf

\begin{tabular}{ll}
\hline KARGER & ( 2003 S. Karger AG, Basel \\
Fax +4161306 1234 & Accessible online at: \\
$\begin{array}{l}\text { E-Mail karger@karger.ch } \\
\text { www.karger.com }\end{array}$ & www.karger.com/oph
\end{tabular}

